\title{
Analysis of the Interaction Design for Mobile TV Applications Based on Multicriteria
}

\author{
Ana Lisse Carvalho, Marilia Mendes, Plácido Pinheiro and Elizabeth Furtado \\ University of Fortaleza (UNIFOR), Graduate in Applied Computer Science (MIA), \\ Av. Washington Soares, 1321 - Fortaleza, Ceará, Brazil \\ \{ana.lisse,marilia\}@edu.unifor.br \{placido,elizabet\}@unifor.br
}

\begin{abstract}
Since mobile TV represents a new paradigm for interaction design, many different candidate solutions of interaction can be possible. There is not yet any work that explores how to consider the users' experience with technology to analyze the best solution(s) to the interaction design of a mobile TV application. This report brings an experience about what criteria addressing users' experience influence designers to make a decision. A qualitative analysis was performed for different solutions and was based on a multicriteria approach. These criteria were classified in accordance with users' preferences and their intentions of use, which were obtained from a ranking modeled with the ZAPROS III multicriteria method. Results revealed great influence of the users' familiarity with applications in the ease of navigation.
\end{abstract}

Keywords: DTV, Mobile Interaction Design, ZAPROS, Multicriteria, HCI

\section{INTRODUCTION}

In domains (as digital TV, smart home, and tangible interfaces) that represent a new paradigm of interactivity, the decision of the most appropriate interaction design solution is a challenge. Researchers of Human-Computer Interaction (HCI) field have promoted in their works the validation of design alternative solutions with users before producing the final solution. Taking into account users satisfaction and their preferences is an action that has also gained ground in these works when designers are analyzing the appropriate solution(s). Recent research reveals that the understanding of subjective user satisfaction is an efficient parameter for evaluating interface [1]. In domain of interaction design for digital TV, we claim that it is necessary to consider both international aspects for supporting the accessibility for all and digital contents for supporting a holistic evaluation (content and user interface) of the TV applications that show a content through their user interfaces.

Structured methods for tasks generally consider quantitative variables (such as: quantity of errors, number of times that the user consulted help, time taken to find a new function, etc). The users generally are encouraged to judge the attractiveness of the interface, and from these comments, evaluators produce qualitative texts [2]. The aesthetic quality of a product influences users' preferences but other qualitative aspects influence judgments that transcend the aesthetic appearance [3]. When dealing

Please use the following format when citing this chapter:

Carvalho, A. I... Mendes, M., Pinheiro, P.. Furtado, F., 2007. in IFIP International Federation for Information Processing. Volume 254, Research and Practical Issues of Enterprise Information Systems II Volume I. eds. L. Xu. Tjoa A., Chaudhry S. (Boston: Springer). pp. 389-394. 
with Digital Television applications, new interface project and evaluation paradigms have been developed, as shown by Angeli [3]. No work however has integrated qualitative criticisms in order to obtain a ranking of interface solutions.

Furthermore understanding subjective questions, another problem deliberated for this research is about traditional means of evaluation. It is quite rigid and not flexible to the emergence of new project alternatives and new ways of considering these alternatives. For example, designers evaluated two interface solutions applying usability tests, and choose one to implement. During a system development, more three design solutions arisen as a result of new usability pattern. How can designers consider these new alternatives? How does evaluate if a new pattern is better than an old solution? At traditional means, usability tests should be applied to all alternatives. With a multicriteria model, these decisions are efficient and only some alternatives would be evaluated. In this project, three interface solutions for Mobile Digital Television Application were evaluated qualitatively by applying verbal decision analysis. This strategy adequately mapped user preferences furnished information which helped to judge solutions for the project. Provide a holistic evaluation of interactions situations and more information to understand and organize subjective questions. A ranking generated by the model is a tool which makes it easy to insert new alternatives and judgments for interfaces.

In order to be able to use the model, hypotheses were elaborated. From these hypotheses, criteria were established as well as usability tests applied in order to obtain information on user preferences. The ZAPROS III method, which belongs to the Verbal Decision Analysis framework, was used [4]. Applying to problems having nature qualitative and difficult to be formalized, called unstructured [5].

\section{HYPOTHESES AND EVALUATION SCENARIO}

The following hypotheses were the basis for elaborating the multi-criteria model which adheres to the reality of evaluations for applications of mobile Digital Television:

- Hypothesis 1: The user's experience with similar types of applications for navigation influences the choice of standard which is easier to use, more exact and more satisfying to the user;

- Hypothesis 2: If the content is interesting, the user will choose the project of his preference;

- Hypothesis 3: The user interacting with the interface while moving, leads him to choose the easiest interface to use;

- Hypothesis 4: Although the user may have experience with a similar interface, he may choose another interface while in movement;

- Hypothesis 5: If access to unattractive content is easy for the user, he tends to prefer the interface with which he has more familiarity.

Once the hypotheses were defined, usability tests with three mobile TVD prototypes were elaborated. The tests served to elicit user preferences. The results of the tests were entered as data for the model. The usability tests were applied with younger users who had wide experience with palms, DTV and desktop computing 
devices. The users evaluated were 12 university students and the duration of the test for each user was between 10 and 20 minutes. Two different locations were used: the usability laboratory and a natural environment (field study). Interface Designers and Usability Specialists were present during the tests. For each user, the test began with a sample portal application for digital TV on a television. This was done so that the user would have knowledge of how the application would work on digital TV.

\section{COMPUTATIONAL RESULTS}

The ZAPROS III can be applied to problems with the following characteristics [4]: the rule of the decision is developed in the form of alternatives; there is a large number of alternatives; evaluations of the alternatives can only be established by humans and not by measuring devices; the graduations of quality inherent to the criteria are verbal definitions that represent the subjective values of the decision maker.

The criteria used in the evaluation of this work were established with the assistance of specialists in the usability of mobile TVD of the Usability and Quality of Software Laboratory. The specialists wished to analyze the aspects that had the greatest influence in the choice of a determined interface project. According to the hypotheses, the following criteria were modeled verbally:

1. Familiarity of the user with a determined technology; if a standard is similar to a determined technology familiar to the user, this standard is preferable to him, since it is easier for him to use.

2. Attractiveness of the task to be carried out; if the standard allows for good visibility of the content, does the user prefer this standard in relation to the standard that has a familiar appearance to the applications that he is used to using?

3. Locomotion of the user during the manipulation of the interface; if the standard allows for good spatial orientation, which doesn't demand much of the user's attention to manipulate, will it be preferred in relation to the standard that has a familiar appearance to the applications that he is used to using, and at the same time allow for excellent viewing of the content?

With conditions thus defined, the ZAPROS III method can be applied according to our following presentation. In table 1 , the values are shown for the criteria directed to the aspects on which the definition of the levels of attractiveness among the standards is based.

The order of preference among the criteria values was established during the application of the tests by means of observation. It was found for example that when the users were moving and trying to execute a task in a determined prototype, they complained that: it was difficult to move and manipulate the prototype at the same time. After the tests, the responses to the questionnaires were collected and interpreted. Questions such as "What prototype did you prefer? And Why?" indicated the order of preference among the project alternatives and also which criteria values were decisive for the choice. The Joint Scale of Quality Variation - JSQV which was gradually elaborated and validated with information from the tests. The scale is then elaborated and it is possible to use the transitive operation [6] to diminish the quantity 
of necessary comparisons. JSQV resulted: $\mathrm{A} 1 \prec \mathrm{A} 2 \prec \mathrm{B} 1 \prec \mathrm{C} 1 \prec \mathrm{C} 2 \prec \mathrm{A} 3 \prec \mathrm{C} 3$

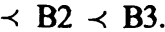

Table 1. Criteria and Associated Values

\begin{tabular}{|c|c|c|}
\hline Representation & Criteria & Values \\
\hline A & $\begin{array}{l}\text { Familiarity of } \\
\text { the user with a } \\
\text { determined } \\
\text { technology }\end{array}$ & $\begin{array}{l}\text { A1. No familiarity is required with similar applications of } \\
\text { determined technology } \\
\text { A2. Requires little user familiarity with applications of } \\
\text { determined technology } \\
\text { A3. Manipulation of the prototype is fairly easy when the } \\
\text { user is familiar with similar applications }\end{array}$ \\
\hline B & $\begin{array}{l}\text { Attractiveness } \\
\text { of the task }\end{array}$ & $\begin{array}{l}\text { B1. Allows high accessibility to the content } \\
\text { B2. Allows medium accessibility to the content } \\
\text { B3. Accessibility to the content is quite difficult }\end{array}$ \\
\hline C & $\begin{array}{l}\text { Locomotion of } \\
\text { the user during } \\
\text { the } \\
\text { manipulation } \\
\text { of the interface }\end{array}$ & $\begin{array}{l}\text { C1. The user was not hindered in any way when } \\
\text { manipulating the prototype while moving } \\
\text { C2. The user was occasionally confused when manipulating } \\
\text { the prototype while moving } \\
\text { C3. The spatial orientation of the application is hindered } \\
\text { when the user is moving }\end{array}$ \\
\hline
\end{tabular}

The next step of the method was to carry out the comparisons of the alternative standards. Each alternative was studied in order to define which criteria values materialized the prototypes. The usability tests also supplied important information on how the users described the standards (for example, the majority of users said that access to content using prototype 3 (three) was quite easy - a criteria value of B1, but that it required a lot of familiarity with Desktop applications - criteria value A3). Finally, the established relationship was: Alternative 1, prototype 1 - A1 B2 C1; Alternative 2, prototype 2 - A2 B2 C1 and Alternative 3, prototype 3 - A3 B1 C2.

Each Quality Variation - QV of JSQV is numbered in ascending order from 1 (one) to 9 (nine). The sum of the determining QV numbers for each alternative is the Formal Index of Quality - FIQ [4]. The calculation for each alternative is presented in table 2 . With the FIQ values, the ranking of the prototypes is organized assuming that the alternative with the lowest FIQ value represents the highest rank and the best alternative. The alternative with the highest FIQ value is the least preferred prototype. Table 2 shows the ranking.

Table 2. FIQ Values for Each Alternative

\begin{tabular}{|c|c|c|c|}
\hline Alternative & QV determinants & FIQ & RANK \\
\hline Prototype 1: A1 B2 C1 & B1 & 3 & 1 \\
\hline Prototype 2: A2 B2 C1 & A1, B1 & $1+3=4$ & 2 \\
\hline Prototype 3: A3 B1 C2 & A3, C1 & $6+4=10$ & 3 \\
\hline
\end{tabular}




\section{DISCUSSION}

The resulting rank and the preference scale between two criteria values prove the validity of the hypothesis: The user's experience with similar types of applications of navigation influences the choice of the standard, which is easier to use, more exact and more satisfying for the user. This influence is a determining factor for the choice of the most preferable project solution. A1 was the value at the top of the scale of criteria values, as Hypothesis 1 demonstrated. The interesting content leads the user to choose the project with which he had the greatest affinity. This affinity is determined by the degree of similarity with applications commonly used by the user. The criteria value $\mathrm{B} 1$ came just after $\mathrm{A} 1$ and $\mathrm{A} 2$, showing that affinity is even more important a determinant than the content accessed, no matter how important or attractive this content is. Then, Hypothesis 2 proved. Considering in detail Hypothesis 3. Since C1 was less preferred than the criteria values for A1 and B1, we observed that when the user is moving and interacting with the interface, he chooses the interface that is easiest to use (that with which he has more affinity and ease of access to more interesting content). Hypothesis 4 was refuted because even when users had some experience with similar interfaces, when they were moving, they chose interfaces according to the criteria of ease of use. This is shown by the order of preference that $\mathrm{C} 3$ is preferable to $\mathrm{B} 2$ and $\mathrm{B} 3$. But the user's experience with similar devices is still a determiner in that we can see that $\mathrm{A} 3$ is preferable to $\mathrm{C} 3$. Hypothesis 5 "If access to unattractive content is easy for the user, he tends to prefer the interface with which he is more familiar," cannot be demonstrated completely with the criteria used up to now. In the current model we can perceive that if the content is attractive or not is less preferred than the influence of experience with similar applications. However, we cannot yet verify that access to unattractive content exercises a different influence than access to more attractive content.

The ranking of the alternatives showed prototype 1, similar to TVD applications, as being the most preferred. Prototype 2, similar to palm applications, with a Formal Index of Quality value very close to that of prototype 1, showed that the difference in attractiveness between the two is quite modest. Prototype 3, similar to desktop applications, proved inadequate for the content of TVD. The very high FIQ value in relation to the two other prototypes shows the low attractiveness for this type of TVD application.

\section{CONCLUSIONS}

A discussion of user-centered development process taking into account real people's needs is described in [3]. On the contrary of this study, that shows an experience which three solutions of design for different devices were defined and analyzed based on how users experience each solution. In [7] the authors describe a multi-criteria approach in which the execution of its steps allows to identify the order of attractiveness of a list of usability patterns for a certain interactive task of DTV applications. In this experiment and using the ZAPROS III approach, a qualitative analysis about the users' preference and their intentions of use with executable 
prototypes could be better appreciated. The standards of usability for mobile Digital Television applications should strongly consider which applications are most used by the target clientele. It proved to have characteristics such as flexibility, such that new project alternatives can be added, allowing researchers to better understand the needs and opinions of the target users of mobile TVD. It even assisted the usability specialists themselves to understand the relationship (order of preference) among the commonly used criteria for the interface project using standards. Research is also being developed to discover how to validate the hypotheses with quantitative metrics. What metrics are possible for each hypothesis? Could these metrics be entry points for information used to elaborate a multicriteria model such as ZAPROS III?

It is important to point out our intention was not to compare navigation techniques (as scrollbars, tap-and-drag, and so on) on mobile devices to identify the best one when users are performing navigation and selection tasks. Our goal was to help designers understand how criteria related to users' experience could influence their preference for a solution. In addition, we showed how to integrate two different areas (HCI and OR - Operational Research) describing an approach for evaluating the Interaction design in a subjective perspective of $O R$. It means researchers interested in making qualitative analysis of the interaction, which leads to more objective results can use this proposal.

\section{REFERENCES}

1. K. Chorianopoulos and D. Spinellis, User interface evaluation of interactive TV: a media studies perspective, Univ Access Inf Soc. Volume 5, (2006), p.209.

2. N. Tractinsky, A.S. Katz, and D. Ikar, What is beautiful is usable, Interacting with Computers. Volume 13, Number 2, pp.127-145, (2000).

3. A. Angeli, A. Sutcliffe, and J. Hartmann, Interaction, Usability and Aesthetics: What Influences Users' Preferences?, in Symposium on Designing Interactive Systems, Proc. of the 6th ACM conference on Designing Interactive systems (2006), pp.271-280.

4. O. Larichev, Ranking Multicriteria Alternatives: The Method ZAPROS III, European Journal of Operational Research. Volume 131, pp.550-558, (2001).

5. H. Simon and A. Newell, Heuristic Problem Solving: The Next Advance in Operations Research, Operation Research. Volume 6, pp.4-10, (1958).

6. O. Larichev and H. Moshkovich, Verbal Decision Analysis for Unstructured Problems (Kluwer Academic Publishers: Boston, 1997).

7. K.S. Sousa, H. Mendonça, and M.E.S. Furtado, Applying a Multi-Criteria Approach for the Selection of Usability Patterns in the Development of DTV Applications, in $I V$ IHC'2006 (Natal, 2006). 